\title{
Carousel of feelings: Reversal theory as a design tool
}

\author{
Fleur A. van Midwoud, Roderick G. Huijgen, Ilaria Scarpellini, Steven F. Fokkinga \\ Delft University of Technology
}

\begin{abstract}
This paper describes a design research project that aimed to find the best ways to convey reversal theory in a holistic way to designers, so that its principles could be applied in creating more effective and compelling products. Over a number of iterations, two games were developed that together can be used as a tool in design processes. This tool incorporated the key insights of the theory and presented these in a format that was suitable for the creative mindset of designers and the challenges they face in the design process. Besides their primary aim, the games also proved useful in understanding the emotions and motivations of user target groups and in communicating the theory to third parties.
\end{abstract}

Keywords: Reversal theory, user experience, product design, design tool, design research

\section{Introduction}

Since its introduction, reversal theory has been successfully applied in practical fields like sports, business and advertising, among many others (Apter, 2013). Recently, this list has been expanded to include product design - the practice of developing the functionality, aesthetics, and experience of all products and systems that are used in everyday life.

The theory has proved especially fruitful in user-centered design, which applies insights about how design impacts people on an emotional, motivational and behavioral level. For example, Gielen (2011) showed how design students used insights of reversal theory to design for children's play and learning. Fokkinga \& Desmet (2012) demonstrated how negative (parapathic) user emotions can enrich the user experience with the use of protective frames. Both projects took a number of connected insights from the theory and showed how these can add a deeper understanding of user experience and lead to more satisfying and successful products.

These projects represent interesting first steps to disclose parts of reversal theory to designers. At the same time, they are limited in scope, because they have not fully done justice to its comprehensive and holistic nature. More than a

Fleur A. van Midwoud, Industrial Design Engineering, Delft University of Technology; Roderick G. Huijgen, Industrial Design Engineering, Delft University of Technology; Ilaria Scarpellini, Industrial Design Engineering, Delft University of Technology; Steven F. Fokkinga, Industrial Design Engineering, Delft University of Technology.

Correspondence concerning this article should be addressed to Fleur A. van Midwoud, Delft University of Technology, Faculty of Industrial Design Engineering, Landbergstraat 15, 2628 CE Delft, The Netherlands E-mail:fleur.vanmidwoud@gmail.com set of insights, reversal theory intends to be a 'grand theory' of psychology - a certain outlook on the whole of human motivation, emotion and behavior (Apter, 2007).

There are several reasons why designers would benefit from understanding the theory in its entirety. First of all, by only being aware of a parts of reversal theory, designers might create product ideas that apply a few of its insights in a way that is contrary to the main ideas behind reversal theory and may thus not be (fully) successful. For instance, a designer could apply the understanding of the telicparatelic distinction to come up with a product for long-term use which reverses the user to the paratelic state, ignoring the fundamental principle that the user is dynamic, and will not always (want to) be in this motivational state. Secondly, several more advanced topics, such as psychodiversity and dynamic personality are difficult to understand without a comprehensive grasp of the theory, while such topics are arguably also some of the most interesting to apply in design. Lastly, the scope and complexity of user-centered design has greatly expanded in the last decades. In the past, designers limited their focus to an understanding of ergonomics (the study of how users related physically to products) and cognitive usability (the study of how users understand to use products). However, as products and users became more demanding, designers are increasingly expected to have a deep understanding of what people feel and want in life. This development requires them to navigate the complexity of human emotion, motivation and behavior.

At the same time, designers are not trained psychologists, and most of them do not have the time or resources to explore and study how insights from psychology can improve their understanding of users. Design research, a field that studies and generates knowledge that supports the design practice (e.g., Stappers, 2009), increasingly invests time to understand psychological theory and create design approaches and 
design tools (e.g., Van Boeijen, Daalhuizen, Zijlstra, \& Van Der Schoor, 2013). This process often involves the following ingredients:

1. Selection: With an abundance of (psychological) knowledge available, an important task for design researchers is to select and test theories that they expect to benefit the design process.

2. Translation: The information should subsequently be translated into a set of insights that show the impact of products on human experience and behavior. These insights should be actionable, meaning that they are concrete and give direction in the design process.

3. Formatting: Although the design practice involves several analytical aspects, it is above all a creative process. No matter how informing a theory or approach is, if it fails to inspire creativity in the designer, it will not be successful.

The format in which the information is presented plays a crucial part in this success. First, the use of ample and fitting examples, narratives, and storyboards is important to gain understanding and empathy with the user. Second, because designers have to actively apply insights into a process that is largely intuitive, tools are often interactive, meaning that designers learn from them by actively doing things. This can simply mean that explanatory text is varied with exercises and thought experiments, but it can also mean that tools contain game elements. Third, the informational format relates to how text and visuals are shaped to engage the designer. Because designers are often visual thinkers, their understanding often relies on visual representations instead of text. Finally, because design processes are as varied as the products they produce - from toothbrushes and desk lamps to cars or smartphones - design tools need to be flexible and versatile, and can never take the form of a cookbook approach.

This paper describes a design research project that generated a toolkit that aimed to disclose reversal theory to designers. The outcome was a set of two games for designers, which are to be played in succession. The first is a board game with pawns and dice, which intends to holistically explain the core principles of reversal theory by giving players control over a fictional character who goes through several reversals and changes in arousal and transactional outcome. The second is a set of cards that designers can use to inspire the design process in the conceptual phase. These games were developed over a number of iterative cycles of development and testing with design students.

The paper first describes the process of developing the toolkit, and then elaborates on the specific iterations. In each iteration, it evaluates how successful the toolkit was in fulfilling its aims. Next, additional uses of the toolkit, besides informing and inspiring designers, are described. Lastly, the paper discusses some limitations and implications of the tool for future research.

\section{Overall method}

The following sections describe the eight iterations that resulted in the final toolkit. Each iteration was characterized by a development phase, in which a new version of the tool was imagined and designed, and a testing phase, in which the new version was tested with design students. The evaluation of the testing phase subsequently led to new requirements for the next development phase, and so on. Some iterations were larger in scope and consisted of major changes in the way insights were conveyed, other, smaller iterations involved fine-tuning of text, visuals, or both. The first three authors undertook both the development and testing phases each of whom has a design background and research training. In each testing phase, the toolkit was offered to invited design students in a session that lasted roughly an hour. The participants were all students pursuing a master's degree, consequently they had already at least four years of experience in design. In this session, one moderator facilitated the design students' efforts to apply the toolkit, while two researchers observed the process from an adjacent room with video facilities (with the exception of the very first session, in which all three researchers were moderating). The participants were encouraged to verbalize their thoughts during the process, which lasted about 45 minutes, while the observers and moderators took notes of the actions and statements of the participant. Specifically, they would observe:

- to what extent the participant seemed to understand the activity;

- to what extent the participant seemed to be engaged by the activity;

- to what extent the examples that the participant generated fitted the insights of the theory;

- to what extent the generated examples were creative and imaginative

After the activity, there was a 15-minute discussion of the process with the participants, the moderator, and the observers (who would then step into the room). The main evaluation points were:

- to what extent participants had understood the insights that the toolkit aimed to convey. This was done by asking participants directly about their own perceived level of understanding, and also by asking whether they could explain specific topics and concepts;

- to what extent participants had felt to be on top of the process - i.e. feeling challenged, but not confused;

- to what extent participants had felt engaged;

- to what extent participants felt the toolkit would help them in their own design process.

The choice to use master's design students as participants was pragmatic, but also fitted the aims of the project. These students already had a few years of experience in designing, but were also very open to try and learn new things and adjust their approach. Several of them had already been exposed to 
other design approaches that incorporated insights from psychology, which allowed them to compare the reversal theory toolkit to other design tools.

\section{Toolkit iterations}

Generally speaking, the development of the toolkit comprised four main stages, each of which consisted of one or more iterations:

1. In the first stage, the authors developed the initial tool by engaging with reversal theory and making a first attempt to translate it to actionable insights. This resulted in a card set that designers could use as a creative tool. The evaluation showed that designers were unable to use the card set in their design activities, because they lacked overall insight of what the theory was about.

2. The evaluation led to the conclusion that another, separate tool was necessary, which would explain the theory to designers before they would make an attempt to apply the insights. This resulted in a board game, in which the player controls a character who goes through a reversal, emotional change, or both in each turn. This board game was evaluated and fine-tuned in several iterations, until it was effective in explaining the core insights of reversal theory.

3 . The card set was re-evaluated and developed for use as the follow-up activity to the board game, in which the insights could be applied to design. Several iterations of card sets were made and tested, until they proved helpful in supporting a creative design process.

4. In the last stage, the board game and card set were combined and designed to fit better together. A final evaluation of the entire tool was done.

In the following sections, each stage and iteration is discussed in more detail. Appendix A shows an overview of all the iterations in terms of toolkit changes and evaluation characteristics.

\section{Stage 1 - Ten insights and the initial card set}

To prepare for the first version of the toolkit, the authors studied reversal theory by reading relevant literature (particularly, Apter, 2007, 2013; Cramer, 2013; Desselles \& Apter, 2013), and by having sessions with designers to discuss potentially interesting and relevant elements for design. Through this process, ten primary insights were formulated, which were thought to be both essential to understand the theory, and readily applicable to design. Appendix B shows an overview of these ten insights. For illustrative purposes, three of the insights are described and explained below.

Insight 1 represents a primary insight in the use of reversal theory in design; it explains the role of design in human experience (in design often called 'user experience', or 'UX'). Figure 1 visualizes that there are three main variables that influence user experience: the momentary meta-motivational state of the user, the events, stimuli and environment that the

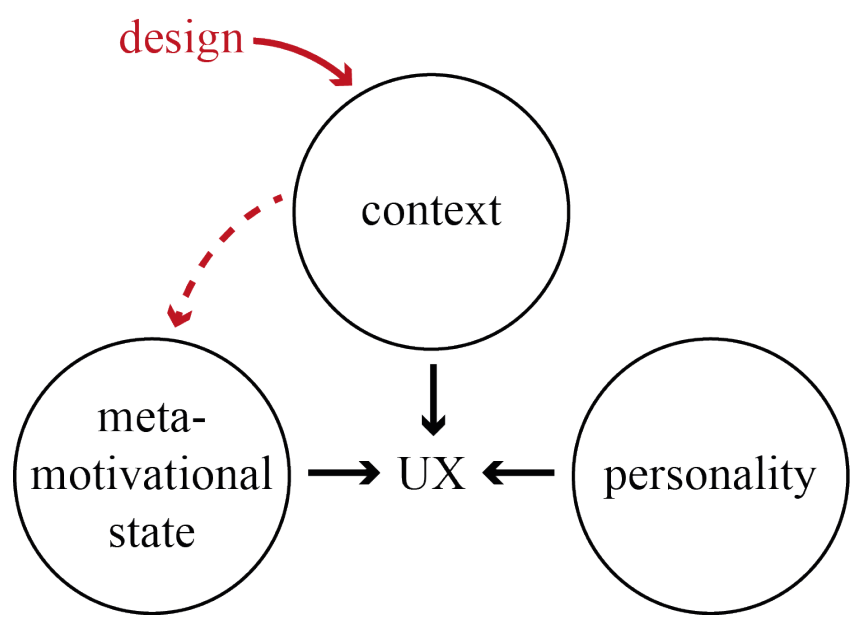

Figure 1. Schematic overview of the relationship of UX with meta-motivational states

user is aware of (context), and the user's personal characteristics, such as what he or she attends to, and his or her state dominance (personality). Design can impact user experience in two ways. It can have direct influence over the physical context, by (re)designing the products or environment of the user. For example, a digital agenda helps to remember appointments, thus decreasing arousal and increasing the pleasure of a user in the telic state. Secondly, design can influence experience indirectly by influencing the metamotivational state. For example, a woman working in an office can reverse to the sympathy state upon noticing a picture of her husband, a contingent event, which is brought about by a product (also see Apter, 2007).

Insight 5 explains how design can make users aware of their motivational states and emotions. In most cases, products will induce reversals, changes in arousal and/or changes in transactional outcome, in a way that the user is not consciously aware of. However, there is also an opportunity to help users get more control over these changes. An example is an office chair that can be quickly adjusted from having a firm and upright angle (suitable for working and the telic state) to becoming tilted and flexible (suitable for the paratelic state). By making explicit that the chair is suitable for these two purposes, users could more easily facilitate the switch from one mental condition to another.

Insight 6 underlines that there is not an ideal, optimal motivational state in which individuals should be all the time. Instead, psychological wellbeing results from experiencing all motivational states over time, with both positive and negative emotional outcomes. This means that when designing for long-lasting experiences, psychodiversity should be one of the main concepts to take into account. For example, work spaces ideally include recreational areas to give employees the opportunity to for breaks that allow them to 
unwind. Without this opportunity, employees would have a hard time reversing to the playful state at the office, negatively influencing their energy levels and productivity.

The card set. The ten insights were visualized, accompanied with examples, and elaborated to a level in which they were expected to be understood by designers without external help. After deliberation, it was decided that a physical set of cards would be the most suitable format to convey these insights to designers. It would be relatively easy to produce, and readily adjustable if needed. A card set could also be flexibly used in different activities: for instance, designers could lay out the cards and be free in picking those that interested them, or, alternatively, they could draw the a few random cards from a deck and be coerced to come up with an idea that represents that combination of insights.

The set of cards was categorized according to the specific topic of reversal theory, e.g., reversals, dynamic personalities, and cognitive synergies (Figure 2). The colors on the cards represented four different layers of information, which allowed designers to vary from more abstract to concrete information. The four categories consisted of:

- Definition cards: these cards contained definitions of the reversal theory concepts. These cards conveyed the more fundamental information, necessary for a deeper understanding of the theory. These cards served as a glossary for the terms mentioned on the other cards.

- Design implication cards: these cards explained the insights on a level that showed the relevance for design. These cards clarified the connections between cards within the same category, and supported the understanding of the theory in relation to the application.

- Tips cards: these cards provided extra information and inspiration to complement the implication cards.

- Rules cards: these cards represented important boundaries that should be taken into account by designers when applying the theory.

Testing of the card set. The initial card set was tested in an hour-long session with three participants (design students), none of who had prior knowledge of reversal theory. Three moderators facilitated the participants in their attempt to master the cards. There was not an explicit goal for the participants in the session, other than trying to understand the theory. Participants were asked to discuss their understanding of the information given by sharing situations that could serve as an example. During the test, the four categories guided the session. First, the definition cards were introduced to create a shared understanding among the participants. As soon as the moderator noticed that the information was understood, the next category was introduced.

Results of card set test. The participants found it difficult to understand most of the information in the card set. One often-mentioned reason for this was the unfamiliarity with the jargon and terms used in the cards. Another reason was the lack of examples about the application of the basics of the theory to design, which instead would help participants realize what they could do with the insights on a practical level. The intention of the activity was that participants would come up with their own examples, but they found this challenging without input from the moderator. The freedom that participants had to mix and match cards made it difficult for them to find meaningful connections between the pieces of information given. The main problem was that the cards highly depended on each other to understand the bigger picture, which meant that participants often got frustrated with individual cards. Furthermore, the variety of information was overwhelming rather than inspiring. Lastly, the lack of a clear goal for the session made it unclear to participants what they were expected to do.

Conclusions of stage 1. The first toolkit proved strikingly unsuccessful, both in communicating the reversal theory insights to designers and inspiring new design ideas. This was caused by several practical issues, most notably the underestimation of how difficult participants found it to understand the terms and see the connection between the insights. For these issues, relatively straightforward solutions could be devised: the inclusion of more elaborate definition cards and more cards with examples, and a clearer connection between the cards, to highlight the relations between insights.

However, the most important conclusion at this point in the process was that it seemed impossible to convey the benefit of the theory for design if explained in parts rather than in a holistic way. Although eventually it would be necessary to present individual insights in the application to design, another step was needed as a precursor. This would first give participants an overview of the theory and its view on human experience and behavior, leaving out for the moment its eventual application to design.

\section{Stage 2 - The board game}

This new tool took the form of a board game. The argument was that the best way to understand the core structure of the theory (the four state oppositions; reversals; arousal and transactional outcome; the sixteen emotions) would be for the participant to construct a narrative of a fictional character who experiences all the possible motivational states and emotions. The idea was that the board game would show a visual overview of all the motivational states and a pawn representing the character. The participant would each turn throw a die to see which change the virtual character would go through. Upon arriving at a new motivational state and/or emotion, the participant would have to come up with a short narrative to illustrate the new state, while also being a logical continuation of the constructed stories up to that point. There was no real means to win or lose the game, although the 'soft goal' of the game was to achieve psychodiversity, by visiting each emotion at least once. 

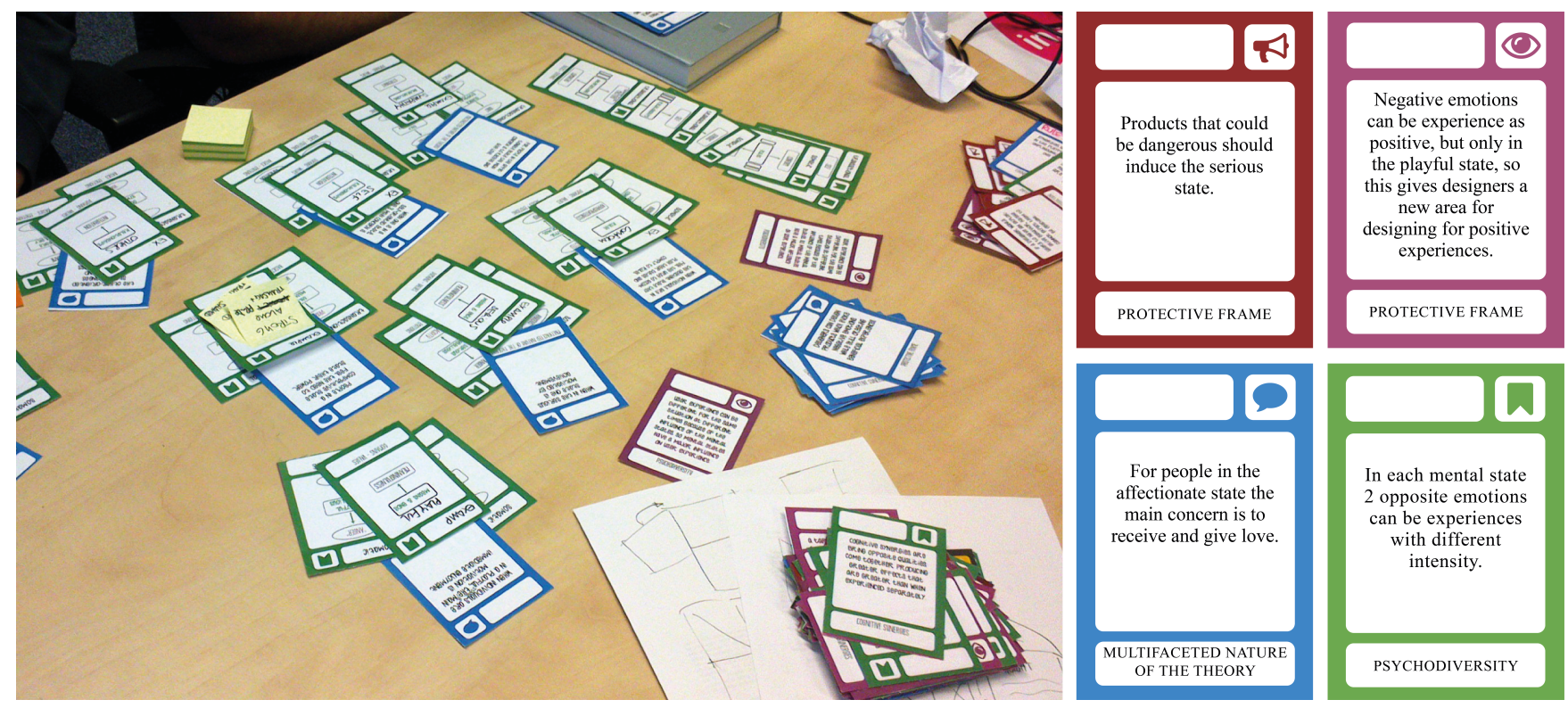

Figure 2. First iteration of the tool

The board game shows two fields, 'pleasure' (somatic emotions) and 'happiness' (transactional emotions). These fields are separated, though connected through the dynamics of the game. The layout and colors show that there are eight motivational states, which each consist of a pair of opposite emotions, one positive and one negative (Figure 3).

Circular dotted arrows represent these levels and the restrictions for emotional change when reversing between motivational states. In the pleasure field, the two rings represent high and low arousal. In the happiness field, the two rings represent a positive or negative transactional outcome.

Dynamics of the board game. The game was played with two players. The players each come up with a fictional character, which is drawn on the character cards. The players were asked to provide some interesting details for their character, such as appearance, occupation, hobbies, etc. Inventing a character together served as icebreaker, and was meant to put players in the mood for role-playing. During the game, the character is represented by two pawns - one in the pleasure (arousal) field and one in the happiness (transactional outcome) field. Through this, players learned that both contribute to the experience of the character. The two separate fields meant to show that while both influence the character, they are not directly correlated. The simplified use of happiness and pleasure avoided having to explain the meaning of somatic and transactional emotions.

The instructions explained that the character has to experience a variety of many motivational states and emotions to attain psychodiversity. Part of this insight was that, although individuals always desire the positive emotions, negative emotions also have to be experienced to fully enjoy the positive ones. Although the ultimate goal was to achieve all sixteen emotions, the game could stop whenever players had enough. In the very first turn, the player would pick a situation card. This helped the players to figure out where to place the pawn, and how the motivational states relate to the context. It also served as the starting point of the story. For example, a situation could be: "entering a plane with luggage, looking for one's seat". To help the players, the card provided the combination of motivational states that resulted from this situation - telic and conforming in the current example. The main part of the game would consist of a number of turns (about 10-20). The turns alternately took place on the somatic and the transactional field. Each turn had a fixed sequence:

1. The players picked a turn card. On this card they started describing what the character want as a result of the current combination of motivational states.

2. The participants rolled the emotion die to see which arousal level (for the somatic field) or which transactional outcome (for the transactional field) they had gotten, and moved the pawn to the corresponding emotion. On the side of the board, the definition of each emotion was presented.

3 . The participants rolled the reversal die to see which motivational state combination would occur (e.g., paratelicconformist, or telic-negativistic), and to move the pawn to that state. There was a one out of four chance that the pawn would stay in the motivational state of the starting point, and a three out of four chance that a reversal would take place.

4. The participants used their imagination to describe on the turn card in words and images what had happened in the 


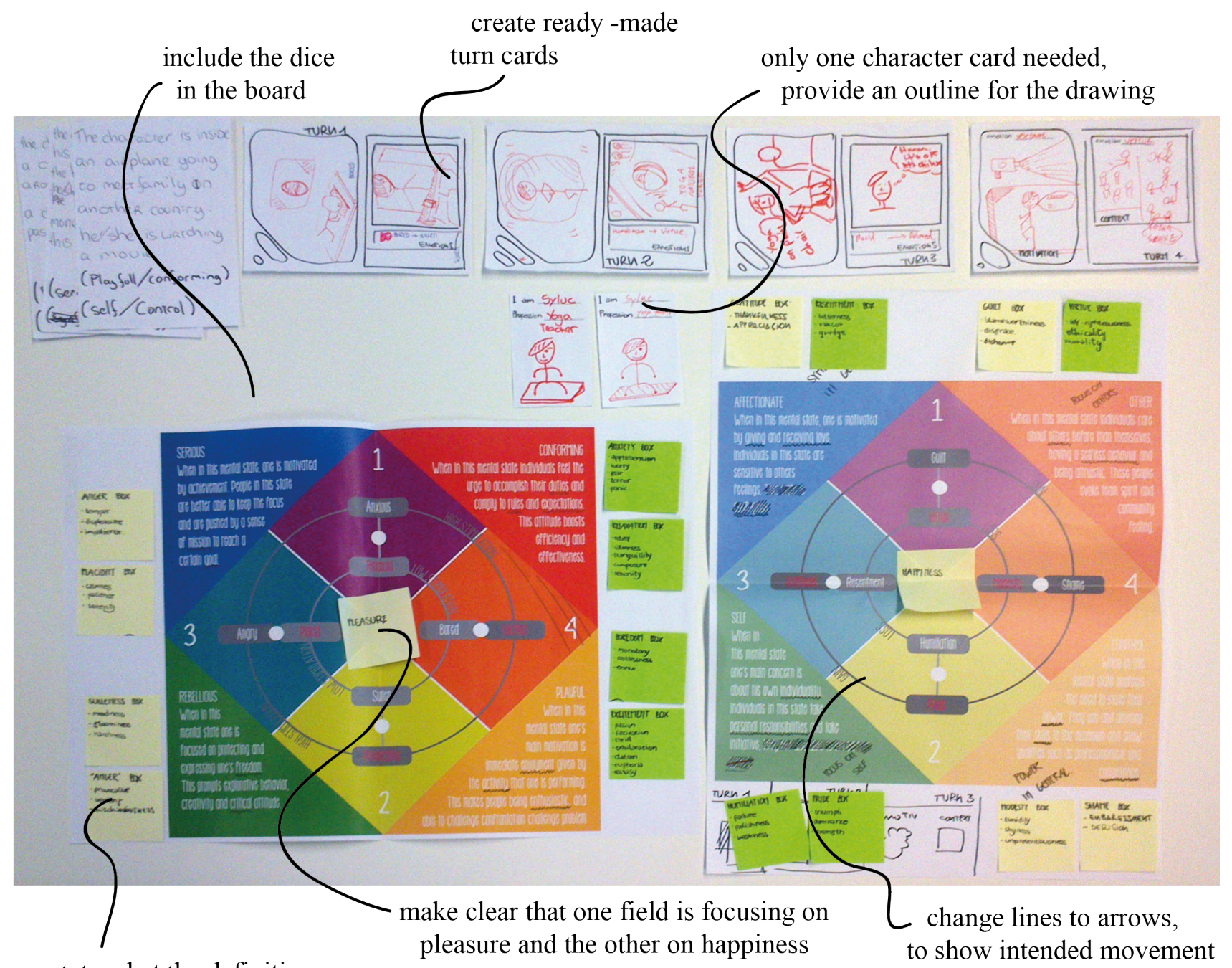

state what the definitions

and synonyms of emotions are

Figure 3. First iteration of the board game

context that induced the reversal and, consequently, the emotion.

Figure 4 shows an example of how the game could start and continue in one turn.

By coming up with examples themselves, instead of offering examples, participants were expected to get a better active understanding of the theory. At the same time, the structure and rules of the board game aimed to prevent that participants would feel lost and confused, as they had in the first iteration of the card set. By playing through several turns, participants ended up with a visual scenario of their character's motivations and emotions (Figure 5). This helped them to understand how dynamic human emotions are, and to what extent these emotions are influence by context.
Testing the first board game. The user test was done with two design students who had no prior knowledge of reversal theory. The two participants were provided with a printed version of the tool, two pawns and the initial card set. The moderator would only intervene when participants got stuck in the game or when they violated one of the game rules,. The test took one hour in total, with an additional fifteen minutes of discussion.

Conclusions of the initial test of the first board game. The game was considered to be fun, even if the participants did not understand it completely. The pleasant and lively mood during the session helped to come up with enjoyable and creative examples and drawings. Being absorbed in the game, participants did not notice time passing by. Although the first few rounds still relied on the moderator to provide 

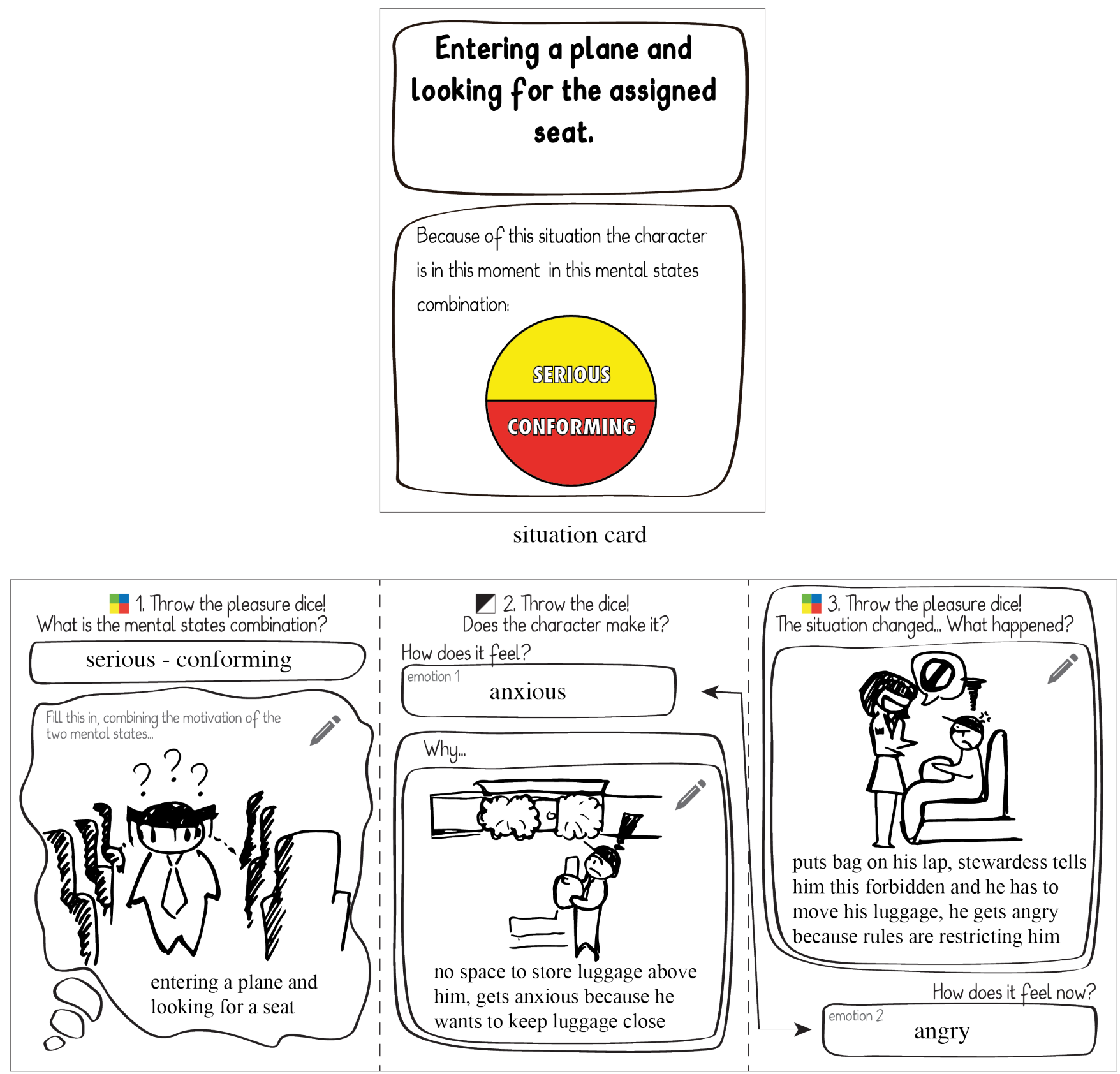

turn card

Figure 4. Examples of the different types of cards

explanations and suggestions, participants were able to continue independently.

The text used to explain the motivational states was found too long and unclear by both participants, and it caused them to read all the text on the board before starting the game, which made the start of the game unexciting. The way that reversals were explained in the game worked well and clear. The participants put the pawns in the right motivational states and emotions, and understood how the reversals induced the emotions. The presence of two separate dice for different purposes was very badly understood by the participants; they had no idea when to throw which die. It seemed that the participants needed visual cues to know what the dice represented and when to roll which die. The introduction of the game did not work as expected and caused major confusion throughout the game. The amount of information at the start 


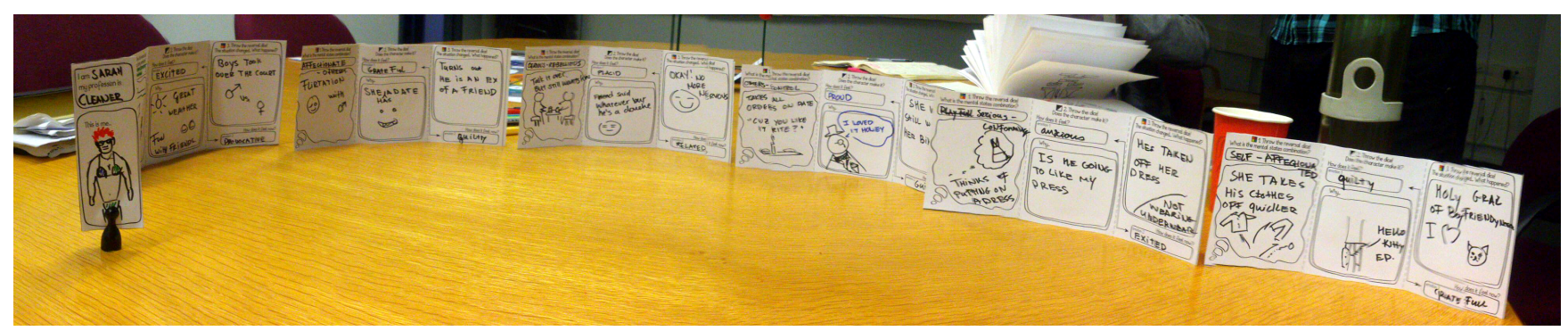

Figure 5. Visual scenario of a characters motivations and emotions

was overwhelming and several parts were forgotten by participants in the course of the game. Several times the players forgot previously explained rules and did not look back to the instructions. It was clear that the provided information should be offered more step-by-step and more supported-byvisuals.

In-between iterations of the board game. In the third stage, the board game was adapted several times before deciding on a final design, both visually and in the dynamics of the game. The visual changes can be found in Figure 6 . Figure 6a shows the first design as described above. Figure $6 \mathrm{~b}$ shows the first redesign of the board game, in which the different pawn locations were clarified, the text on the board was reduced, and the colors were overhauled. Figure $6 c$ shows the changes after testing the redesign, the overall style has been simplified and the board now identifies the two circles as either "pleasure" or "happiness". Figure $6 \mathrm{~d}$ is the final design of the board and is described in the following sections. These decisions were motivated by testing results, user feedback, and design guidelines about how to structure visual information in relation to the role that each graphic element plays in achieving the right use of the board.

Final design of the board game: The carousel of feelings. The focus of the last redesign was to improve the usability of the game (Figure 7). The challenge was to better facilitate the players in understanding how to start the game, and how to keep playing without getting confused. Icons on the board were intended to clarify which die belongs to which field. Similarly, the turn cards received a specific place on the board to clearly indicate which field they belong to. For each motivational state, the motivation was briefly described in a few words by stating the most prominent characteristics, in order to condense the information and to allow for faster reading.

The accompanying manual now featured an introduction of the theoretical basis of the game, which included the core principles of reversal theory and how it related to the game and the design process. The steps in the instructions were similar to previous iterations, but they now included an example to provide guidance and support during the player's first turn. Someone who saw the board game mentioned that it looked like 'a carousel of feelings', which was liked so much that it became the title of the game. The authors felt that it expresses movement, colorfulness, and something that is constantly changing.

\section{Stage 3 - Second part of the toolkit: the card set revisited}

Now that the board game had evolved to a point where it was successful in conveying the principles and thinking behind reversal theory to designers, the task was to work on the part of the tool that would help designers to turn the theory into actionable insights for idea generation. The choice was to reuse the form factor of the first iteration; the card set.

The resulting set of card contained examples, insights, and several tips and rules (Figure 8). The cards were much more visual than the initial set, featuring cartoons that specifically show one example or insight. This made the cards look more attractive and the examples easier to understand and remember.

Dynamics of the card set. The cards were organized into six categories representing the six most concrete and applicable insights that emerged from the study of reversal theory. These were insights number 1, 3, 4, 5, 9, and 10 from the original ten insights (see table 1). The other four insights had already been covered in the board game.

An instruction sheet provided a brief introduction to how the cards should be used and why. The cards included visual guidelines with a style similar to the board game to make the users feel familiar with the provided information. A legend was included to help interpret the symbols on the cards and to distinguish examples, insights, tips, and rules cards on a visual and informational level.

Testing the design. The same participants who had previously tested an early version of the board game were asked to explore the insights cards and to come up with examples that illustrated the insight of the specific categories. Afterwards, they were given five minutes to use the insights to come up with ideas for an imaginary design case. The design case was kept simple to help designers to focus on concrete problems, which allowed for an easy flow of ideas.

Results and conclusions of the initial tests. The information provided on the insight cards did not work well for the participants. They had trouble with the language, the 


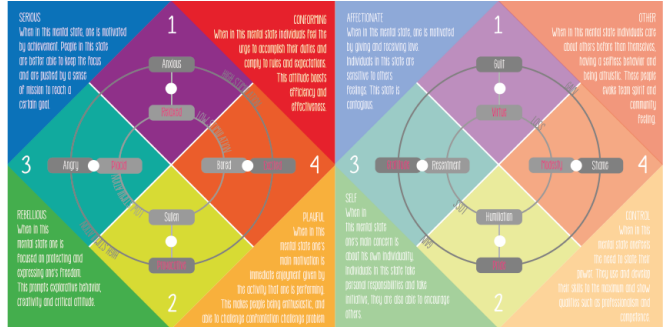

a.

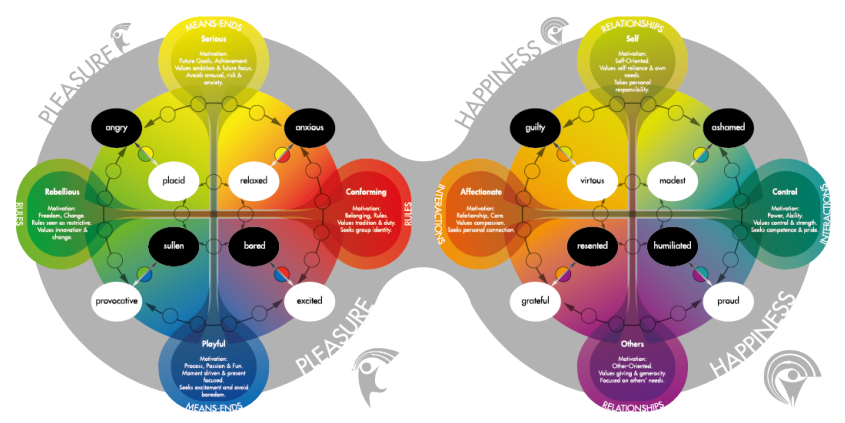

c.

Figure 6. The four iterations of the board game

amount of information, and the complicated terms. Part of the problem was that the level of difficulty was not the same across cards. Because participants picked random cards, they sometimes ended up with more complicated ones in the beginning, which proved frustrating. In addition, the examples were unclear and took a long time to understand. The participants spent much time discussing the examples with the moderator to better understand them, which was in principle fine, but also caused the conversation to become more about the examples than about the insights.

Final design of the card set. The final cards set consists of ten cards, divided over four categories. The categories serve as an indication of the card's topic (Figure 7). Subtitles were added to identify the specific content of each card. A number of changes were made from the last iteration. A difficulty scale was introduced, which identified for each card the level of complexity. This gave people the chance, if they desired so, to use the simpler cards first. In addition, some of the language on the cards was made more explicit and concrete and the examples revised to be as clear as possible.

\section{Stage 4 - Validation of the Carousel of Feelings}

In the fourth and final stage, the results from the second stage (the board game) and the third stage (the card set) were combined for use in a single session. The games were tested

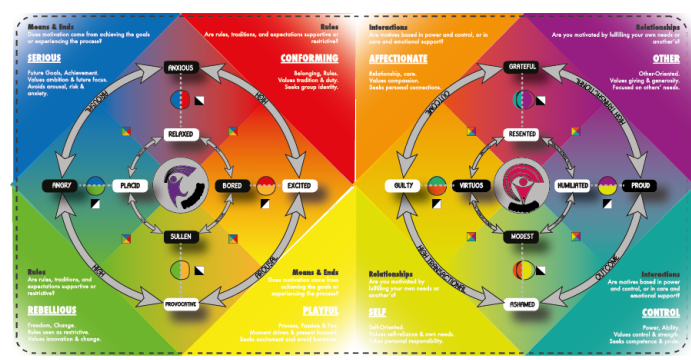

b.

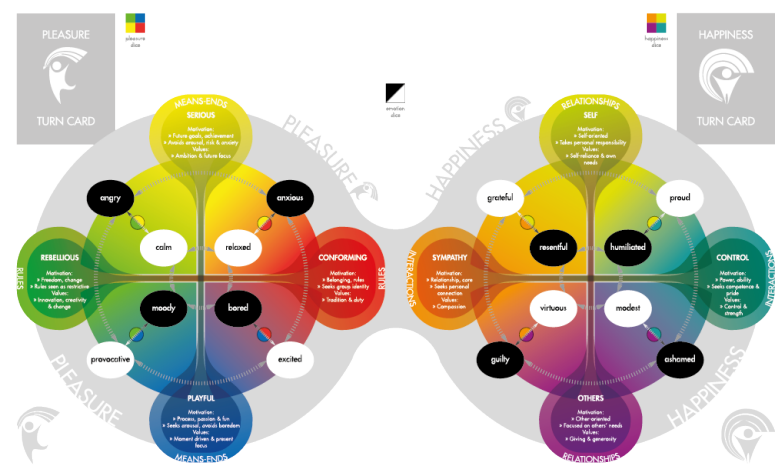

d.

with design students who had not previously been exposed to earlier versions of the game or to reversal theory. The outcomes of the card set session depended on the understanding generated during the board game session, so the participants had over two hours for the complete test. The participants had 45 minutes to play the board game, followed by a 30minute break to let the information sink in, and concluded with 45 minutes of using the card set. The session ended with a discussion and a final evaluation of the tool.

Discussion of the final test. Overall, the participants truly enjoyed the first part of the tool. The start of the game still moved slowly and featured a steep learning curve, but after a few turns the game became enjoyable and dynamic. Participants noted that they were amazed by how far the story could continue. It also became clear that the more fun the participants had, the better and more imaginative the results became, which were not always the most realistic ones. Moreover, the playful mood made the overall process faster because the participants were more comfortable and not afraid to make mistakes. It was surprising that many participants did not attempt to read the instructions completely beforehand and as a result got into difficulties when playing the game. This made the first turns take unnecessarily long to finish and while the participants struggled the moderator could only refer them to the instructions. 


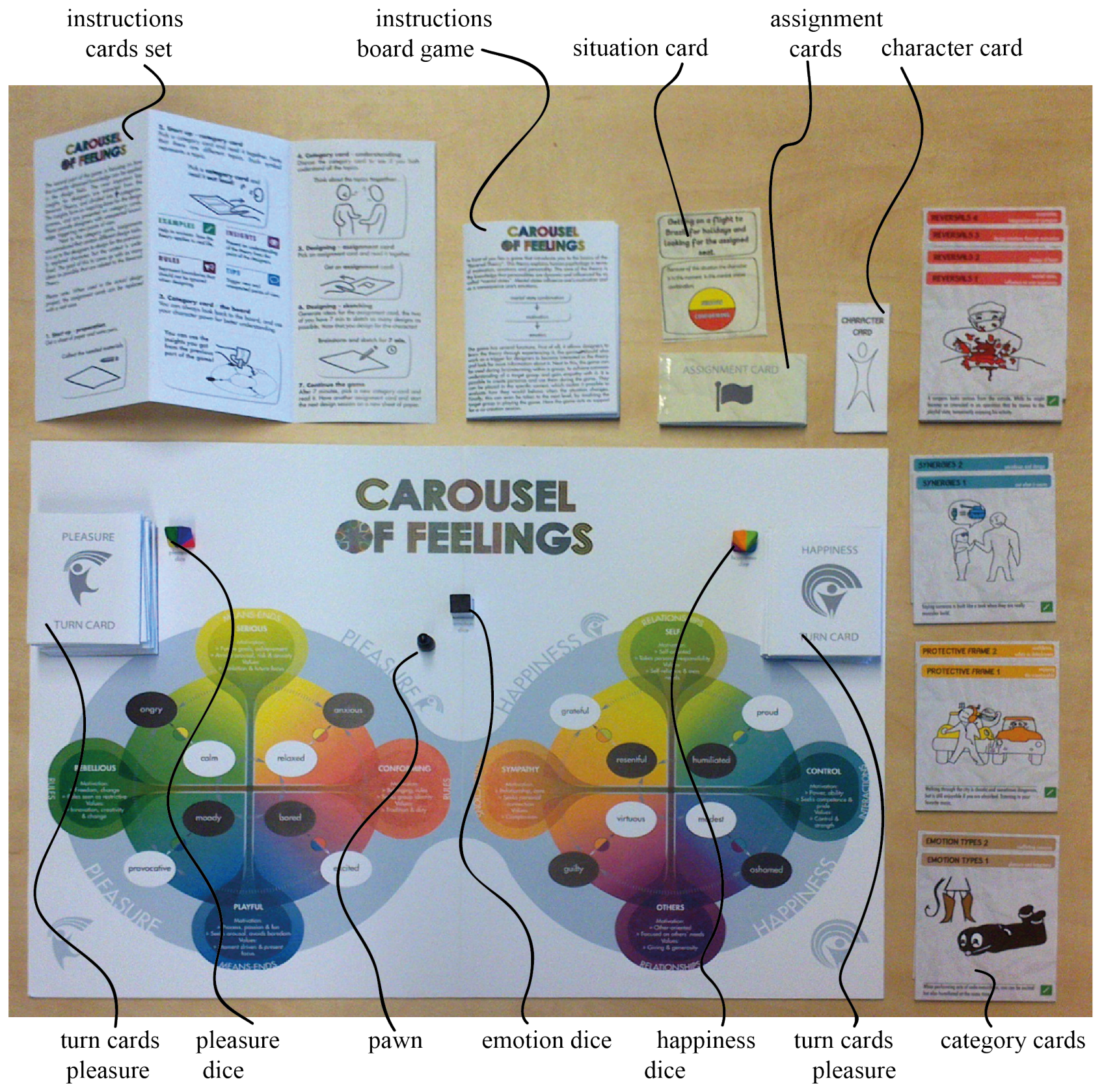

Figure 7. The Carousel of Feelings

The longer the participants played the game, the better they grasped the ideas behind the theory. Unfortunately, this process could not be extended beyond the 45 minutes that was allocated for the board game. When the participants were questioned after the test to check their understanding of the theory, most participants were able to explain what motivational states were and how they influence experience, what reversals and how they can be used, and how the board game could be used as a tool for design (both as a generative and reflective tool).

Most of the insight cards were understood well and the participants could discuss the theory and start using it in a design case. However, the seven minutes time limit for each idea turned out to be very short for getting inspiring ideas. Sometimes, participants seemed to overthink the information and were reluctant to simply come up with first ideas. This 


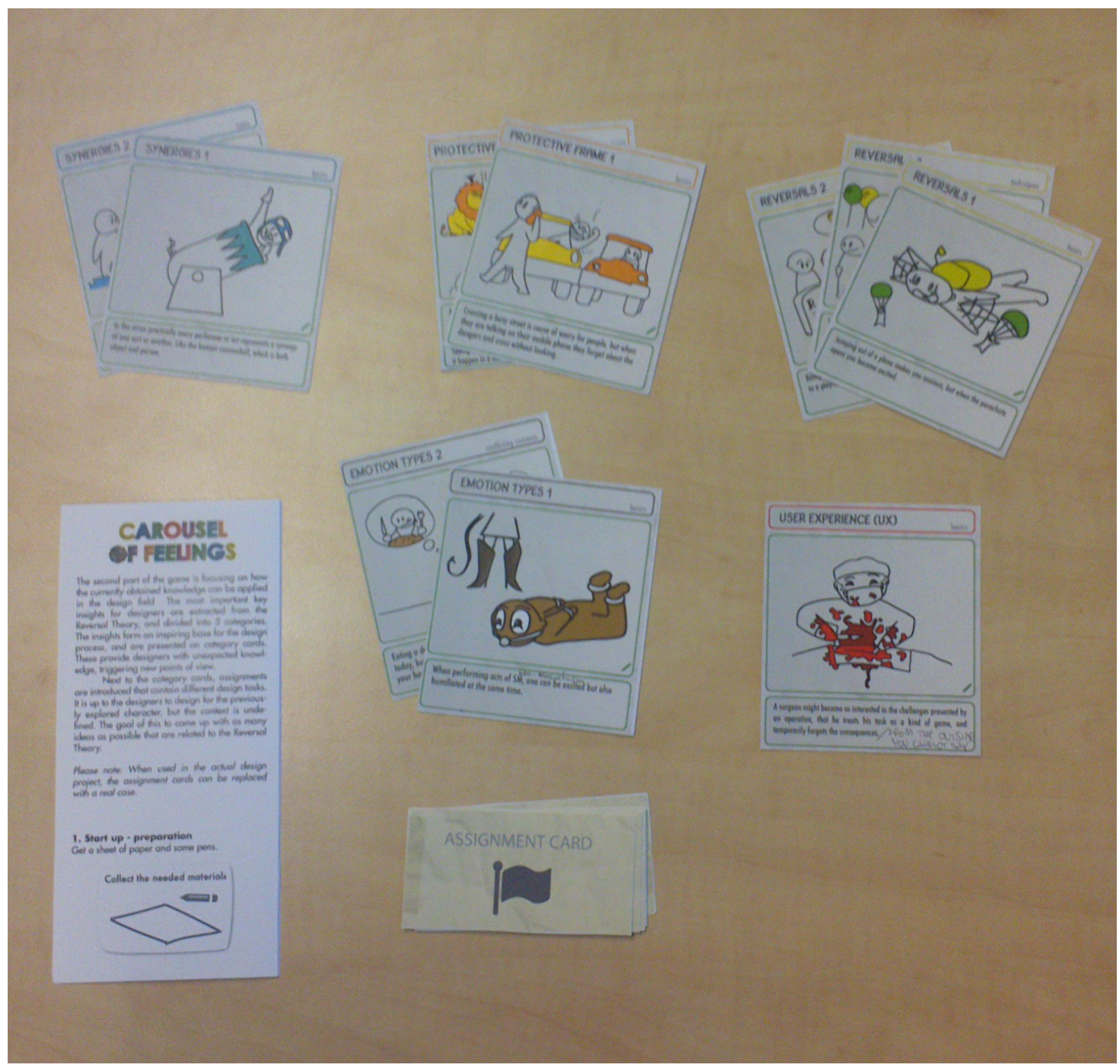

Figure 8. The iteration of the card set

was partly due to the amount of information on the cards and the fact that participants were not yet comfortable with all the provided insights. The cards further increased their interest in the theory; most of the participants wanted to learn more about the theory because they saw the potential for their own work.

A general conclusion was that the presence of a knowledgeable moderator remained essential, at least in the current iteration of the tool. Especially participants who are new to reversal theory and the game would not be able to independently play the games and acquire the intended insights. First of all, the moderator would provide guidance and additional explanation in the early stages of the game and occasional validation of the examples generated by the participants along the way. Furthermore, the moderator had to encourage participants to be in the right state of mind while playing the game, to boost enjoyment and creativity. 


\section{Additional applications of the tool}

During and after development of the toolkit, it became clear that the games could have additional applications besides informing and supporting designers. The first application was to explain reversal theory to third parties, such as clients, to help them better understand how reversal theory was included in a project and to feel more ownership over the process. The second application was to play the game with the intended end-users within a design project, in order to gain a better understanding of their daily reality and how the context in which they live and work influences their motivational state and emotions. For both these additional applications the board game worked best because it was the more engaging of the two games and not specifically geared towards designers.

\section{The game as a way of communicating a reversal-theory approach to a wider audience}

The game was tested during a presentation for twenty flight experts from the airline KLM (the Dutch national airline). Because the group was bigger than the intended amount of players, the game was slightly adjusted. Instead of using one game board, several were provided, while the authors used a bigger version in front of the group to demonstrate the steps that had to be taken. The game itself became an interactive group act, in which everyone who had a good idea could propose an example that illustrated a turn. It was sometimes challenging to get a high level of creativity in the examples, compared to tests with designers. But as the game progressed, more and more participants came up with creative examples. It was striking that a large number of examples were (partially) based on situations that were actually experienced with passengers during a flight. The game provided them with an approach to better analyze and understand recalled situations in terms of the emotions and reactions of passengers. More importantly, they understood how they could respond to better handle similar situations in the future.

\section{The game as a way to better understand the target group and target context}

After the game had been developed, it was used in one of the subsequent design cases in order to better understand the user target group - flight attendants. In a generative session with six flight attendants, the tool was used to imagine possible emotions and motivational changes within the context of airplane travel. With the tool the flight attendants had clear instructions on how to proceed in the conversation and the flow of information could be moderated by the designer. Because of the combination of a short presentation and the tool, the theory was easily understood by the participants. They were able to think in terms of motivational states and reversals and project these on the situations within the cabin and their personal experience. Because the group was relatively large for one game board and because they all turned out to be very expressive, there was an abundance of contextually relevant information; so much, in fact, that the moderator was unable to keep up with the information. Therefore, a group of three or four participants would be more ideal in a setup like this.

\section{General discussion}

This paper described a project that investigated ways in which reversal theory could be communicated to designers and that developed a set of two games to fulfill this aim. We see several opportunities for the games as they were developed. The later tests with design students show that the games are successful in conveying a basic understanding of the reversal theory insights in designers. Moreover, they were enthusiastic about applying the insights in their work. After playing the games, several students asked where they could read more about the theory.

Secondly, the application of the games could potentially reach further than the design domain, and also help to inform people in fields like business, sports and marketing about reversal theory. Furthermore, it could be used in psychology courses or in schools to explain the basics of reversal theory in an engaging way. The board game is expected to be especially effective in this regard. The approachability and flexibility of the game make it easy to adapt it to any specific context. Indeed, it could even be used with individuals who are interested in understanding their own motivations and emotions better.

There are still a number of improvements that could be made in the games. For instance, it will take more testing to find the correct balance in the amount of participants and the amount of results. Similarly, there is not yet an optimum balance between the time that is spent playing the board game, and time spent designing with the card set. Secondly, the visual design of the game, which features a dynamic shape and playful colors, is intended to emphasize the enjoyment that players should have while playing the game. However, this form factor and even the fact that it is a game, might be a cause for some people to not recognize its serious intent. As it has become clear that the game is only successful if participants fully engage in it, this might become a reason that it will not work for everyone. During the tests some participants showed signs of being in the telic state, which conflicted with the lack of a clear goal and ending to the game, but also had a negative effect on the creativity of the participants. One of the possible reasons for this state in the participants was their insecurity about their drawing skills, which they were asked to use to sketch the situations of the character. This was sometimes solved by having the mod- 
erator do most of the visualizations, but this was also a bit much for the moderator to take on in addition to his or her other duties. Lastly, the games could be made available so others can use it in their research or practice. This may mean that some adjustments have to be made to make it accessible for a broader audience. The current game has to be printed and assembled by the user. This effort might be a too high threshold for using the game. It would be more user-friendly to distribute the game readymade, although this would come with additional costs.

The game was briefly tested by attendees of the reversal theory conference in Reims, France, in 2013, where it gathered interest. It would be interesting to further test the game with reversal theory experts to assess the validity of the game. For future research, it would also be interesting to evaluate the effectiveness of the games when used by designers in a full design project. All the user tests described in this paper were executed with design students in sessions lasting only a few hours and unrelated to their own design projects. In order to validate its full potential, it would be insightful to study how designers use the game during a complete design case, for example, within a design education assignment.

\section{References}

Apter, Michael J. (2007). Reversal theory: the dynamics of motivation, emotion, and personality (2nd ed.). Oxford: Oneworld.
Apter, Michael J. (2013). Developing reversal theory: Some suggestions for future research. Journal of Motivation, Emotion, and Personality, 1(1), 1-8.

Cramer, Kenneth M. (2013). Six criteria of a viable theory: Putting Reversal Theory to the test. Journal of Motivation, Emotion, and Personality. Vol, 1, 9-16.

Desselles, Mitzi L., \& Apter, Michael J. (2013). Manipulating Motivational States: A Review. Journal of Motivation, Emotion, and Personality, 1, 44-49.

Fokkinga, Steven F., \& Desmet, Pieter M.A. (2012). Darker Shades of Joy: The Role of Negative Emotion in Rich Product Experiences. Design Issues, 28(4), 42-56.

Gielen, Mathieu A. (2011). Designing for the real child Toy designers and psychologists in dialogue on reversal theory. Paper presented at the The 4th conference of the International Association of Societies of Design Research, Delft, The Netherlands.

Stappers, Pieter Jan. (2009). Meta-levels in Design Research: Clarifying the Roles we play in Design, Research, and Elsewhere. Paper presented at the International Association of Societies of Design Research: Rigor and Relevance in Design, Seoul, South Korea.

Van Boeijen, Annemiek G.C., Daalhuizen, Japp J., Zijlstra, Jelle J.M., \& Van Der Schoor, Roos S.A. (2013). Delft Design Guide. Amsterdam: BIS publishers. 
CAROUSEL OF FEELINGS

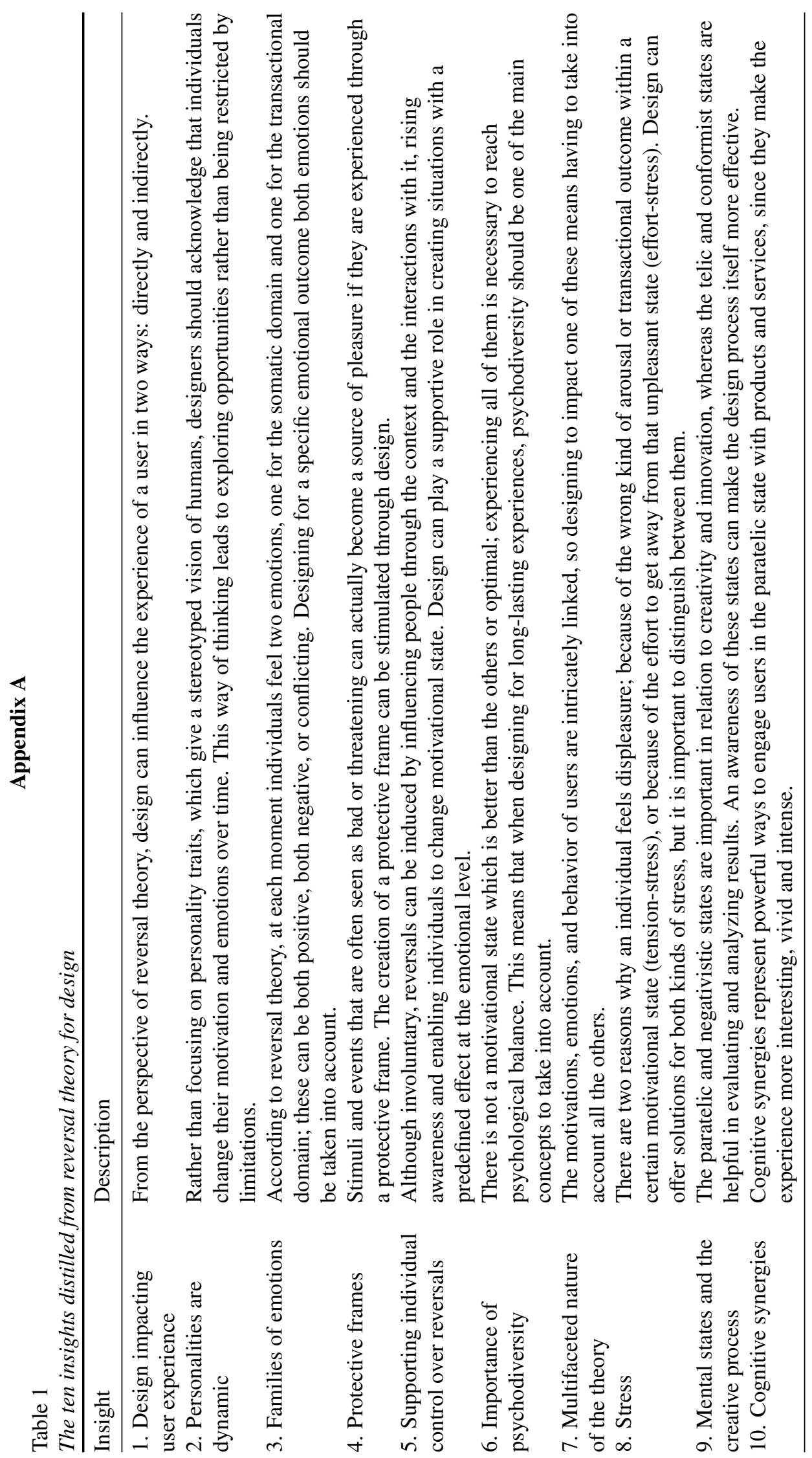




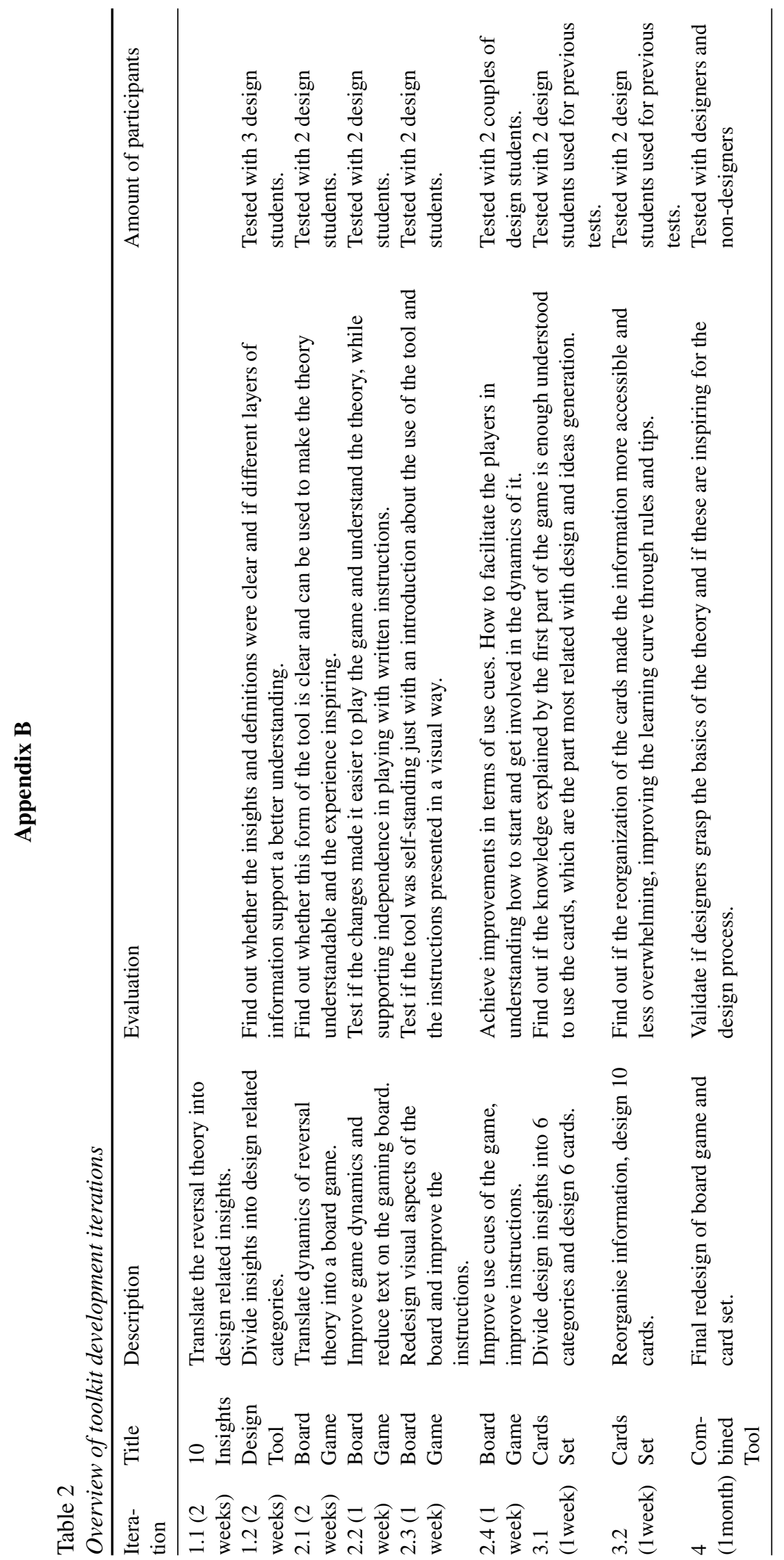

